\title{
Capital social, relaciones sociales y bienes públicos: ¿qué conexiones?
}

\author{
Social capital, social relations and public assets: \\ what are the connections?
}

PIERPAOLO DONATI

UNIVERSITÀ DI BOLOGNA

Artículo recibido: 24 de abril de 2018

Solicitud de revisión: 6 de junio de 2018

Artículo aceptado: 3 de septiembre de 2018

Donati, Pierpaolo (2018). Capital social, relaciones sociales y bienes públicos: ¿Qué conexiones? Recerca. Revista de Pensament i Analisi, 23, pp. 169-192.

Resumen

Toda investigación empírica muestra que el capital social es un factor esencial para producir y preservar los bienes públicos, pero las teorías difieren en cuanto al papel desempeñado por las relaciones sociales. Algunos piensan que los bienes públicos no necesitan redes sociales, mientras que otros argumentan que, sin redes sociales, el capital social no se crea y, por lo tanto, los bienes públicos no se valoran. El presente trabajo mantiene que la comparación entre estas teorías opuestas, o al menos divergentes, solo se puede resolver demostrando si, o no, y bajo qué condiciones, las redes de relaciones sociales producen el valor social añadido (VSA) que se traduce en el capital social necesario para apoyar bienes públicos.Adoptando la perspectiva de la sociología relacional podemos ver y medir el valor social añadido de las relaciones sociales en redes primarias y secundarias, conduciendo a la emergencia de los bienes públicos.

Palabras clave: capital social, bienes relacionales, valor social añadido, relaciones sociales, redes sociales, sociología relacional.

\section{Abstract}

Although empirical research reveals that social capital is an essential factor to produce and preserve public assets, theories differ as to the role that social relations play. Some believe that public assets do not require social networks, while others argue that social capital without social networks cannot be created and, therefore, public assets are not valued.This paper mantains that a comparison between these opposing, or at least diverging, theories can only be made by demonstrating whether, and under what conditions, social relation networks produce social added value (SAV) or not, which becomes the social capital needed to support public assets. From the relational sociology perspective, we can see and 
measure the SAV of social relations in primary and secondary networks that lead to public assets emerging.

Key words: social capital, relational assets, social added value, social relations, social networks, relational sociology.

\section{LA CUESTIÓN: ¿CÓMO SE GENERA EL CAPITAL SOCIAL QUE VALORA LOS BIENES PÚBLICOS?}

En el campo de las ciencias sociales, toda la investigación empírica insiste en que el capital social (CS) da valor a los bienes públicos ${ }^{1}$, pero las teorías sociológicas se encuentran divididas entre dos grandes corrientes de pensamiento. Por un lado, los académicos que apoyan la tesis de que los bienes públicos dependen esencialmente de la cultura cívica y que las redes sociales son un producto indirecto y no estrictamente necesario para la promoción de los bienes públicos, la cual tiene lugar aparte de las redes sociales. Por otro lado, los académicos que sostienen que esa promoción depende necesariamente de las redes sociales, y que, de hecho, consiste precisamente en la creación de redes sociales dotadas, como veremos, de ciertas cualidades y propiedades.

Bajo esta división subyace una concepción diferente y opuesta de la relación social. Para el primer grupo de académicos la relación social no añade ningún valor social, puesto que el capital social consiste, en su opinión, en un legado cultural -un espíritu cívico o algo similar- que sostiene en mayor o menor grado la producción de bienes públicos, en tanto que este legado cultural ha sido internalizado e incorporado por los individuos en un contexto geográfico concreto sin ninguna necesidad de una relación específica entre ellos. Para el segundo grupo de académicos, en contraste, el capital social se basa, precisamente, en las relaciones sociales, que generan el valor social añadido que constituye -o tal vez, «instituye»- los bienes públicos no estatales. Clarificar esta cuestión requiere, por lo tanto, que nos preguntemos si existe realmente el valor social añadido de las relaciones sociales o no.Y si existe, cuál es y cómo podemos medirlo. Responder a estas preguntas es crucial para observar el capital social y sus beneficios

1 En esta contribución, el concepto de público se usa en el sentido clásico. Marco Tullio Cicerone, en su tratado político De re publica (I, 25,39), afirma que «La res publica es una cosa del pueblo; y el pueblo no es cualquier agregado de personas, sino un grupo de asociados que comparten los derechos para la protección de sus propios intereses». Por lo tanto, el término "público» no está relacionado necesariamente con el sistema político del estado, sino que se refiere a todas las formas comunitarias de la vida social que trabajan para el bien común. 
como una verdadera forma de capital -como Fragkandreas (2012) ha argumentado recientemente.

La tesis de la sociología relacional afirma que el capital social genera y da valor a los bienes públicos mediante el valor social añadido de las relaciones. El capital social y los bienes públicos relacionales son dos realidades que se generan y se regeneran mutuamente, o, por el contrario, que se suprimen. Debemos abandonar marcos teóricos circulares y recursivos y concebir las relaciones entre capital social y bienes públicos relacionales como procesos morfogenéticos que tienen lugar en el tiempo. Cuando adoptamos esta perspectiva, podemos observar el valor social añadido (VSA) de las relaciones primarias (interpersonales) y secundarias (asociaciones más allá de la relación personal).

En mi opinión, el valor social añadido es, precisamente, el efecto que surge de la reflexividad agente, interactiva y sistémica de los vínculos sociales cuando -si y sólo si- son considerados y puestos en práctica como oportunidades y medios de enriquecimiento -y, por tanto, como fuentes de revalorización- en lugar de considerarlos como medios de coacción y opresión que inhiben a los actores sociales. Aquellos que rechazan que el capital social tiene una naturaleza relacional, sólo pueden observar su presencia o su ausencia, su mayor o menor eficacia, pero no pueden explicar cómo y por qué se genera o se disuelve. Sin embargo, aquellos que adoptan una perspectiva relacional pueden dar cuenta de los procesos generadores del capital social y de los diferentes beneficios que éste produce en función del valor social añadido de las relaciones que lo constituyen.

\section{ES NECESARIO ENTENDER LA NATURALEZA RELACIONAL DEL CAPITAL SOCIAL}

2.1. ¿Las redes sociales ayudan o son un obstáculo para promover los bienes públicos?

Muchos académicos que han estudiado el capital social han llegado a la conclusión, o bien de manera explícita (Banfield, 1958) o bien de manera implícita (Putnam, 1993, 2000; Cartocci, 2007), de que las redes sociales informales -primarias o de proximidad- y las redes sociales formales -secundarias, o lo que es lo mismo, de asociaciones organizadas- tienden a facilitar la cerrazón de los individuos y su adhesión exclusiva a intereses personales o de una comunidad concreta. Las actitudes de apertura hacia 
un «otro» generalizado -votar, otorgar confianza, donar sangre, etc.-, en cambio, aparecerían, por parte de la sociedad civil, para favorecer la producción de externalidades para el contexto social completo; produciendo así beneficios públicos.

La consecuencia más significativa de este modo de ver el capital social es la elusión o, al menos, la desestimación, de la importancia de las relaciones sociales, ya que las redes de interacción social se excluyen del estudio tanto del capital social como de los bienes públicos que éste produce. En resumen, parece que la valorización de los bienes públicos a través del capital social no necesita de relaciones sociales con características específicas y poderes propios. Por ejemplo, el politólogo italiano Cartocci (2007) sostiene que (1) el capital social no tiene conexiones con el concepto de red social; y (2) que las redes de proximidad establecen relaciones negativas con el capital social y con el espíritu cívico de los individuos, puesto que, en su opinión, las redes sociales sólo promueven intereses particulares, de cabildeo y de la mafia.

Estas dos tesis, sin embargo, han sido cuestionadas por una serie de investigaciones teóricas y empíricas que han probado exactamente lo contrario. La primera de estas tesis conlleva pasar por alto un gran número de estudios sociológicos ${ }^{2}$ que subrayan la profunda e ineludible conexión entre el concepto de red social y el de capital social. La segunda tesis ha sido refutada por numerosos estudios teóricos y empíricos que han arrojado luz sobre las condiciones bajo las cuales las relaciones de proximidad resultan ser eficaces a la hora de aumentar la pro-socialidad y la orientación de los individuos hacia un «otro» generalizado ${ }^{3}$.

En concreto, un estudio realizado en Italia mostró la existencia de una continuidad entre capital social primario, secundario y generalizado (Donati \& Tronca, 2008). La definición de estos diferentes tipos de capital social se observa en la Figura 1, que también aspira a clarificar la terminología utilizada en las subsiguientes aportaciones. Esta investigación descubrió que, por un lado, el capital social familiar y el capital social comunitario están correlacionados positivamente con el capital social asociativo y el capital social generalizado, $y$, por otro, que estos trabajan conjuntamente en la valorización de los bienes públicos. La relativa continuidad de las diferentes formas de capital social indica que existen entre ellas interdepen-

2 Véase Ballet \& Guillon (2003), Bidart (1997), Bogarti et al. (1998), Bourdieu (1980,1985), Burt (1995, 2000, 2001, 2005), Coleman (1988, 1990), Degenne \& Forsé (2003), Lin (1999, 2000, 2001a, 2001b). Para el contexto italiano véase: Bagnasco et al. (2001), Amaturo (2003), Barbieri (1997, 2003, 2005), Chiesi (1999, 2005).

3 Véase Donati (ed. 2003), Donati \& Colozzi (2006), Wollebaeck \& Selle (2002), Uslaner (2002). 
dencias significativas y sinergias recíprocas. Pero esto sólo se puede observar si se adopta una perspectiva relacional del análisis sociológico. ${ }^{4}$

Figura 1

La distinción entre las diferentes formas de capital social (CS): primario, secundario y generalizado

\begin{tabular}{|c|c|c|c|}
\hline $\begin{array}{l}\text { Diferentes } \\
\text { tipos de CS: }\end{array}$ & $\begin{array}{l}\text { Esfera social } \\
\text { (o sujeto): }\end{array}$ & $\begin{array}{l}\text { En qué se basa el CS } \\
\text { (sus dimensiones): }\end{array}$ & $\begin{array}{l}\text { CS específico de esa } \\
\text { esfera produce: }\end{array}$ \\
\hline $\begin{array}{l}\text { Capital } \\
\text { social (CS) } \\
\text { Primario }\end{array}$ & $\begin{array}{l}\text { a) CS familiar } \\
\text { b) CS paren- } \\
\text { tal } \\
\text { c) CS comu- } \\
\text { nitario } \\
\text { de redes } \\
\text { informa- } \\
\text { les (de } \\
\text { vecinos, } \\
\text { amigos, } \\
\text { colegas) }\end{array}$ & $\begin{array}{l}\text { 1. Confianza primaria } \\
\text { (cara-a-cara e } \\
\text { intersubjetiva) } \\
\text { 2. Reciprocidad } \\
\text { interpersonal como } \\
\text { un intercambio } \\
\text { simbólico; o el } \\
\text { regalo como un acto } \\
\text { inscrito en un circuito } \\
\text { de intercambios } \\
\text { recíprocos de dar- } \\
\text { recibir-intercambiar } \\
\text { sin equivalentes } \\
\text { monetarios. }\end{array}$ & $\begin{array}{l}\text { Civismo: } \\
\text { El CS produce relaciones } \\
\text { que son cívicas, en } \\
\text { cuanto se basan en las } \\
\text { buenas formas y en la } \\
\text { consideración positiva } \\
\text { del otro, que es el } \\
\text { término de referencia } \\
\text { para la cooperación y } \\
\text { la solidaridad recíproca } \\
\text { en las relaciones } \\
\text { interpersonales. }\end{array}$ \\
\hline $\begin{array}{l}\text { Capital } \\
\text { social (CS) } \\
\text { Secundario }\end{array}$ & $\begin{array}{l}\text { CS asociati- } \\
\text { vo de redes } \\
\text { sociales or- } \\
\text { ganizadas en } \\
\text { asociaciones } \\
\text { de sociedad } \\
\text { civil. }\end{array}$ & $\begin{array}{l}\text { 1. Confianza secundaria } \\
\text { (hacia otros individuos } \\
\text { que tienen en común } \\
\text { la pertenencia a una } \\
\text { misma asociación } \\
\text { política o civil o } \\
\text { comunidad). } \\
\text { 2. Reciprocidad social } \\
\text { expandida (extensión } \\
\text { del intercambio } \\
\text { simbólico hacia } \\
\text { aquellos que } \\
\text { pertenecen a una } \\
\text { misma asociación } \\
\text { civil o política o } \\
\text { comunidad). }\end{array}$ & $\begin{array}{l}\text { Democracia asociativa: } \\
\text { El CS genera formas } \\
\text { asociativas para perseguir } \\
\text { objetivos que no se } \\
\text { pueden conseguir de } \\
\text { manera individual, sino } \\
\text { que requieren confianza y } \\
\text { espíritu de colaboración } \\
\text { entre personas o } \\
\text { grupos sociales que se } \\
\text { reconocen mutuamente } \\
\text { como miembros } \\
\text { de una misma red } \\
\text { social, asociación u } \\
\text { organización. }\end{array}$ \\
\hline
\end{tabular}

4 El diseño de la investigación y las reglas del análisis relacional se ilustran en Donati (2006). 


\begin{tabular}{|c|c|c|c|}
\hline $\begin{array}{l}\text { Diferentes } \\
\text { tipos de CS: }\end{array}$ & $\begin{array}{l}\text { Esfera social } \\
\text { (o sujeto): }\end{array}$ & $\begin{array}{l}\text { En qué se basa el CS } \\
\text { (sus dimensiones): }\end{array}$ & $\begin{array}{l}\text { CS específico de esa } \\
\text { esfera produce: }\end{array}$ \\
\hline $\begin{array}{l}\text { Capital } \\
\text { social (CS) } \\
\text { generaliza- } \\
\text { do }\end{array}$ & $\begin{array}{l}\text { CS en la esfe- } \\
\text { ra del espa- } \\
\text { cio público, } \\
\text { o en la arena } \\
\text { impersonal } \\
\text { del territorio } \\
\text { considerado } \\
\text { como comu- } \\
\text { nidad políti- } \\
\text { ca (multicul- } \\
\text { tural, multiét- } \\
\text { nica, etc.) }\end{array}$ & $\begin{array}{l}\text { 1. Confianza genérica } \\
\text { (generalizada) en el } \\
\text { Otro; esto es, en el } \\
\text { extraño, que uno se } \\
\text { encuentra en la esfera } \\
\text { pública. } \\
\text { 2. Disposición a } \\
\text { colaborar para } \\
\text { producir un } \\
\text { bien colectivo } \\
\text { (la cooperación } \\
\text { se muestra en } \\
\text { la participación } \\
\text { electoral, en los } \\
\text { actos de donación a } \\
\text { organizaciones con } \\
\text { objetivos pro-sociales, } \\
\text { apoyo de iniciativas } \\
\text { que promueven el } \\
\text { bien público, defensa } \\
\text { de los derechos } \\
\text { colectivos, etc.) }\end{array}$ & $\begin{array}{l}\text { Cultura cívica (o espíritu } \\
\text { cívico) en un contexto } \\
\text { local, nacional o global: } \\
\text { el CS genera un espíritu } \\
\text { cívico (o una cultura } \\
\text { cívica) en cuanto } \\
\text { consiste en el ejercicio } \\
\text { de las virtudes del } \\
\text { «buen ciudadano», que } \\
\text { se compromete con, y } \\
\text { se hace responsable de, } \\
\text { los bienes públicos, con } \\
\text { confianza y espíritu de } \\
\text { colaboración en lugares } \\
\text { y esferas de relaciones } \\
\text { impersonales entre } \\
\text { simples conciudadanos } \\
\text { que se reconocen como } \\
\text { miembros de una misma } \\
\text { comunidad política. }\end{array}$ \\
\hline
\end{tabular}

Podemos interpretar el balance de poder entre el CS primario y el CS secundario -tal como se definen en la Figura 1- desde un punto de vista evolutivo -en términos de extensión e importancia- en los varios tipos de sociedad que han existido a lo largo de la historia. En las sociedades segmentarias -aquellas que son primitivas o simples-, el CS primario es alto mientras que el CS secundario es bajo, ya que no existe una esfera social en el sentido asociativo fuera del ámbito familiar y de parentesco -no hay distinción o, de haberla, es muy leve- entre la esfera pública y privada. En las sociedades estratificadas, el poder del CS primario persiste, pero de una forma diversificada según clases sociales - por lo tanto, en un grado medio-alto- a la par que el CS comienza a desarrollarse fuera de las redes familiares y de parentesco. En la sociedad de la modernidad temprana, el CS primario se debilita de acuerdo a la privatización de la familia burguesa y la difusión a gran escala de la familia proletaria, mientras que el CS secundario se incrementa -la sociedad civil burguesa. En sociedades con alto nivel de modernización, en comparación con las 
configuraciones anteriores, se hace patente una reducción tanto del CS primario como del CS secundario debido a un elevado nivel de fragmentación, aislamiento y anomia social. Por consiguiente, lo que se ganó en términos de incremento de CS secundario al dejar atrás las sociedades tradicionales estratificadas según clases y adentrarse en el primer periodo de la modernización, se perdió posteriormente porque, si bien el capitalismo al principio fomenta el CS secundario, después lo erosiona junto con las redes familiares y de parentesco.

En muchos países europeos el debilitamiento del CS tiene que ver con el hecho de que, por un lado, la estructura moderna de mercado ha colonizado las esferas de la autonomía social que eran típicas de la sociedad civil premoderna (habría que pensar en los grupos asociativos que surgieron durante la Edad Media, el Renacimiento y la Ilustración) y de que, por otro, la misma estructura de mercado ha debilitado la bases familiares y subvertido poco a poco el aporte de CS primario en CS secundario. Las variaciones en el CS entre las áreas geográficas altamente modernizadas -centro-norte de Europa- y las áreas modernizadas que aún mantienen fuertes elementos tradicionales -centro-sur de Europa- pueden explicarse desde estas tendencias variables de configuración. Los países del Mediterráneo requieren una discusión aparte, ya que existe entre ellos una gran cantidad de sociedades del tipo que he llamado segmentario y estratificado.

Ante esto, surgen varias preguntas al respecto. Por ejemplo, si es posible pensar en dar forma a una nueva sociedad civil, capaz de perseguir los bienes públicos, mediante el aumento del CS primario y secundario, especialmente en esos países en los que el CS ha ido despareciendo, así como qué tipo de ajustes sociales pueden fomentar una nueva sociedad civil de este tipo. Para responder a estas cuestiones, una red de investigadores ha llevado a cabo diversos estudios sobre las relaciones entre el CS primario y el CS secundario y la relación entre estos dos tipos de CS y otras esferas, como la esfera política de las instituciones democráticas y la esfera económica del mercado. ${ }^{5}$ Los resultados de estas investigaciones han demostrado que los bienes públicos adquieren valor del CS primario, secundario y generalizado.

5 Véase Donati \& Colozzi (2006, 2007), Donati \& Tronca (2008), Rossi y Boccacin (2006a, 2006b). 


\section{2. ¿El capital social es una variable independiente o dependiente?}

Una de las aporías más evidentes en los estudios del capital social consiste, tal como diría Portes (1998), en el hecho de que no está claro si y cuándo el CS es una variable independiente o dependiente. De este modo, uno acaba cayendo en una visión «circular» de los elementos en juego, generando sospecha y confusión sobre la realidad del CS como un factor autónomo dotado de sus propias cualidades y capacidades. No está claro si el CS genera bienes públicos o si las cosas suceden en el sentido contrario, de tal forma que es la existencia de bienes públicos la que genera el CS. Como indicó Portes (1998: 19),

[...] como una propiedad de las comunidades y las naciones, más que de los individuos, el capital social es, al mismo tiempo, una causa y un efecto. Conduce a resultados positivos, como el crecimiento económico y el descenso de crímenes, pero su existencia también se deduce de esos mismos resultados. Las ciudades que están bien gobernadas y que crecen económicamente están en esta situación porque tienen un capital social considerable; las ciudades más pobres tienen una menor cantidad de esta virtud cívica.

Una posible solución a estas dificultades, es acercarse al CS partiendo de una perspectiva relacional que, desde un distintivo punto de vista sociológico, (i) evita los persistentes malentendidos adjudicándolos a los sesgos y distorsiones de los enfoques individualistas y holísticos; (ii) permite que uno vea los bienes públicos -en tanto que generados de una manera relacional- evitando los reduccionismos; en particular, el reduccionismo psicológico -que considera el bien relacional como afectividad, expresividad, etc.- y el reduccionismo económico -que considera el bien relacional como la dimensión humana de las transacciones económicas que ofrece las mejores ventajas competitivas y la mayor utilidad y satisfacción en las preferencias económicas.

Para encontrar una salida a la circularidad de la que he hablado, es útil hacer uso de una noción concreta de «bienes relacionales», como una forma correlativa en la que el CS existe en tanto que se presenta en forma de bien público. Un bien público que se entiende aquí como todo aquel bien que es accesible a cualquiera que quiera tomar parte en él, teniendo en cuenta las normas y condiciones que éste impone para que pueda generarse y disfrutarse conjuntamente con otros.

Los bienes relacionales son un tema que, introducido en la literatura hace más de veinte años, ha padecido y aún padece de las mismas aporías que el concepto de capital social. El concepto fue inicialmente propuesto por Donati (1989) y, simultánea e independientemente, por Carole Uhlaner 
(1989). Estos autores usaron dos enfoques completamente diferentes. Donati ha elaborado el concepto de bien relacional sobre la base de su paradigma de sociología relacional (Donati 2011). Uhlaner, en cambio, lo propuso -y recientemente lo aplicó (Uhlaner 2014)- sobre la base del paradigma individualista de la rational choice, aunque ampliando sus márgenes para poder incluir los componentes expresivos del comportamiento humano en la formación de movimientos sociales.

A partir de ese momento, el concepto de bien relacional fue fagocitado y utilizado por muchos autores, quienes lo aplicaron usando o bien alguno de los dos enfoques mencionados o bien otros enfoques y disciplinas diferentes. Por ejemplo, mientras algunos autores han seguido el enfoque de la sociología relacional (Colozzi 2005, 2006) o el de Uhlaner, enriqueciéndolo con elementos psicológicos (Gui \& Sugden 2005), otros lo han basado en la teoría de juegos (Sacco e Vanin 2000; Sacco \& Zamagni 2006) o lo han reformulado en términos religiosos de comunión y fraternidad (Bruni 2005).

Con los años el concepto de bien relacional ha sido objeto de interés en diversas disciplinas, como en la filosofía moral y en el desarrollo de las comunidades locales, hasta convertirse en sinónimo de «encuentros de valor» entre personas. Becchetti, Pelloni y Rossetti (2008), por ejemplo, definen los bienes relacionales como «(...) el lado afectivo/expresivo, no instrumental, de las relaciones interpersonales». Esta vasta literatura ha hecho que este concepto sea cada vez más polivalente y multidimensional (Donati \& Calvo 2014).

No obstante, en mi opinión muchas de las investigaciones sobre bienes relacionales, especialmente las contribuciones provenientes de economistas y psicólogos, no explican la naturaleza propiamente relacional del bien del que estamos hablando. Especialmente, porque califican el bien para ciertas cualidades y propiedades causales de la acción. individual (como el afecto personal y la empatía), o incluso factores colectivos (como la cultura cívica), en lugar de verlo como un efecto emergente de las relaciones entre agentes.

Ahora bien, a pesar de estas diferencias, existe un consenso creciente sobre la importancia social, económica y política de los bienes relacionales. Su relevancia radica en el hecho de que son producidos por redes sociales que operan sobre la base de principios de reciprocidad y de procedimientos asociativos democráticos (Abad Montesinos \& Abad Montesinos 2014). Se trata de bienes que desde hace tiempo han sido eliminados e incluso reprimidos por la sociedad capitalista, así como por sociedades dominadas por una dictadura, que hoy emergen como la sal de una nueva 
sociedad civil y una democracia avanzada. Estos se crean donde las relaciones entre los miembros son libres y responsables (no restringidas por reglas o poderes de autoridad), no jerárquicas (incluso asimétricas), y no mercantiles o, en cualquier caso, no dictadas por la búsqueda de un beneficio individual (Donati \& Solci 2011).

En esta contribución, apoyo la tesis de que los bienes relacionales, incluso si son intangibles (intangible goods), tienen su propia realidad ontológica. Estos se caracterizan por poseer ciertas propiedades: (i) consisten en relaciones sociales, que no son meras interacciones o transacciones; (ii) estas relaciones sociales son un efecto emergente con respecto a las contribuciones dadas por los sujetos en relación; (iii) estas relaciones tienen una realidad sui generis, es decir, tienen una cierta estructura, que es dinámica y susceptible a los procesos de cambio; (iv) son producidos y disfrutados juntos por aquellos que participan, pero tienen externalidades positivas para la comunidad que los rodea; y (v) la relación generada es portadora de beneficios tanto para los participantes como para quienes comparten sus reflejos desde el exterior, sin que ningún sujeto pueda apropiarse de ellos por sí mismo.

Tomemos, por ejemplo, el bien relacional de la confianza. Nace entre dos o más sujetos que estabilizan una relación que está estructurada por expectativas de confianza mutua, dentro de las cuales tienen lugar interacciones y transacciones que les reportan beneficios y que, sin embargo, también pueden generar un clima de confianza a su alrededor. La confianza interpersonal primaria se puede extender a un grupo más grande, y también se puede proyectar en una asociación u organización más amplia (confianza secundaria), hasta el punto de convertirse en una confianza generalizada. Sin embargo, lo opuesto también puede suceder. Siempre es necesario verificar si, cuando se extiende más allá de la red primaria, la confianza en los demás se mantiene o se debilita y tal vez se convierte en desconfianza. Lo mismo ocurre con todos los bienes relacionales, como la amistad, la cooperación, el intercambio simbólico, incluidos los bienes comunes del llamado capital cognitivo (Ramis Olivos 2014), y muchos otros bienes creados en las redes sociales en Internet.

Conceptos tales como la confianza, la cooperación, la reciprocidad (que son bienes relacionales) son constitutivos del capital social, de ahí que surge el problema de saber si es el capital social el que genera bienes relacionales o viceversa. Ante esto, recientemente ha emergido una nueva discusión sobre los bienes que llamamos relacionales y sus relaciones con el capital social (Donati \& Solci, 2011).Al respecto, cabe destacar que exis- 
ten varias semánticas para ambos conceptos. El concepto de bien relacional se utiliza como explanans y como explanandum en muchas disciplinas -de la economía a la psicología, de la sociología a las ciencias políticas-, exactamente como sucede con el concepto de capital social. Esto indica que existe algún tipo de conexión profunda entre los dos conceptos y que la solución al problema se encuentra en observar estas conexiones.

\section{LAS RESPUESTAS DE LA SOCIOLOGÍA RELACIONAL A TRAVÉS DEL ANÁLISIS DE LOS PROCESOS MORFOGENÉTICOS}

La red de investigación que he dirigido ha demostrado que el enfoque relacional es, precisamente, el enfoque capaz de explicar las relaciones existentes entre el capital social y los bienes relacionales, al mismo tiempo que evita las ambigüedades de los académicos que a veces los utilizan como un explanans y otras veces los utilizan como un explanandum. La solución reside en verlos dentro de una secuencia no circular, sino morfogenética.

Para aclarar este punto, propongo un esquema -Figura 2- que sintetiza las tesis subyacentes a este artículo, según las cuales el CS es producto de los bienes relacionales y, a su vez, es regenerador de los bienes relacionales. La recurrencia entre el capital social (CS) y los bienes relacionales (BR) sólo es aparente, en el sentido de que puede resolverse introduciendo un esquema morfogenético, que tiene en cuenta las fases temporales y la aportación autónoma - «estratificada»- de cada elemento en cada una de las fases del proceso. En este esquema -Figura 2- las relaciones entre CS y BR se observan claramente -habiendo sido simplificadas al máximo: si es el CS el que genera los BR o viceversa, depende de la fase en la que observemos del proceso social como un proceso de morfogénesis social (Archer, 2013). Veamos un ejemplo.

- Tiempo T1: El proceso se inicia cuando una intervención -una acción entre diferentes sujetos- se organiza activando/movilizando la relación/las relaciones entre los actores como un bien que hay que perseguir, como un bien en sí mismo; esto es, como un bien relacional. Por ejemplo, cuando hay que organizar una intervención asistencial -un servicio para cuidado de niños o un centro de cuidado para los ancianos, se diseña y se implementa movilizando las relaciones entre los individuos que van a ser atendidos y los actores de las redes 
primarias y secundarias implicadas. Esto genera una situación de la que puede emerger más o menos, o incluso ningún, CS en términos de confianza, cooperación y reciprocidad entre los actores.

- Tiempo T2 - T3: El hecho de que se genere más o menos CS depende de los actores y de la red que se ha creado y movilizado. Aquí entra en juego la reflexividad de los actores y de sus redes.

- Tiempo T4: Si en la segunda fase del proceso la dinámica generó CS, entonces el bien relacional inicial se regenera o incluso se aumenta. Mientras que, si en la segunda fase del proceso el CS sólo se consumió o se destruyó, no se producen bienes relacionales o incluso desaparecen los bienes relacionales iniciales.

- A partir del tiempo T4 comenzará un nuevo ciclo morfogenético: la red existente de relaciones en el tiempo T4 tendrá que lidiar con las interacciones entre los sujetos, por lo que se volverá a poner en juego de nuevo el CS que puede salir fortalecido o debilitado de ellas.

Es importante destacar aquí que, en la fase interactiva, la reflexividad personal de los actores y la reflexividad relacional de sus redes juegan un rol decisivo, mientras que el contexto estructural tiene su impacto impulsando un cierto tipo de reflexividad más que otro (esto se considera reflexividad o, mejor dicho, reflectividad sistémica (Donati, 2010).

Este marco teórico, por tanto, evita las confusiones entre BR y CS, manteniendo la distinción, pero también la relación, entre ellos.

Por consiguiente, podemos hablar de un VSA del CS que (i) consiste en la (re)generación -más que en el consumo, la destrucción, etc.- de los bienes relacionales; $y$ (ii) puede medirse con la capacidad de la red asociativa para producir relaciones internas y externas que actúan de una forma reflexiva, de tal modo que las relaciones compartidas se vuelven más eficaces; esto es, que incrementan la eficacia operativa de la red -fortalecimiento del requerimiento de lograr objetivos aplicado a la red asociativa (Donati, 2010).

Este es, por tanto, el VSA de las relaciones que llamamos CS, en tanto que la capacidad de general bienes públicos relacionales partiendo desde un contexto organizado con vistas a producir bienes relacionales. En una intervención social que funciona correctamente (porque realmente produce bienes públicos), no hay confusión circular entre el CS y los bienes relacionales; más bien se desarrolla un proceso morfogenético que sigue una precisa secuencia temporal. 
Figura 2

Valor social añadido del capital social (CS) como una (re)generación de los bienes relacionales (BR) en el tiempo (ciclo T1-T4). Esto es, como modificación del orden de las relaciones mediante el orden de las interacciones.

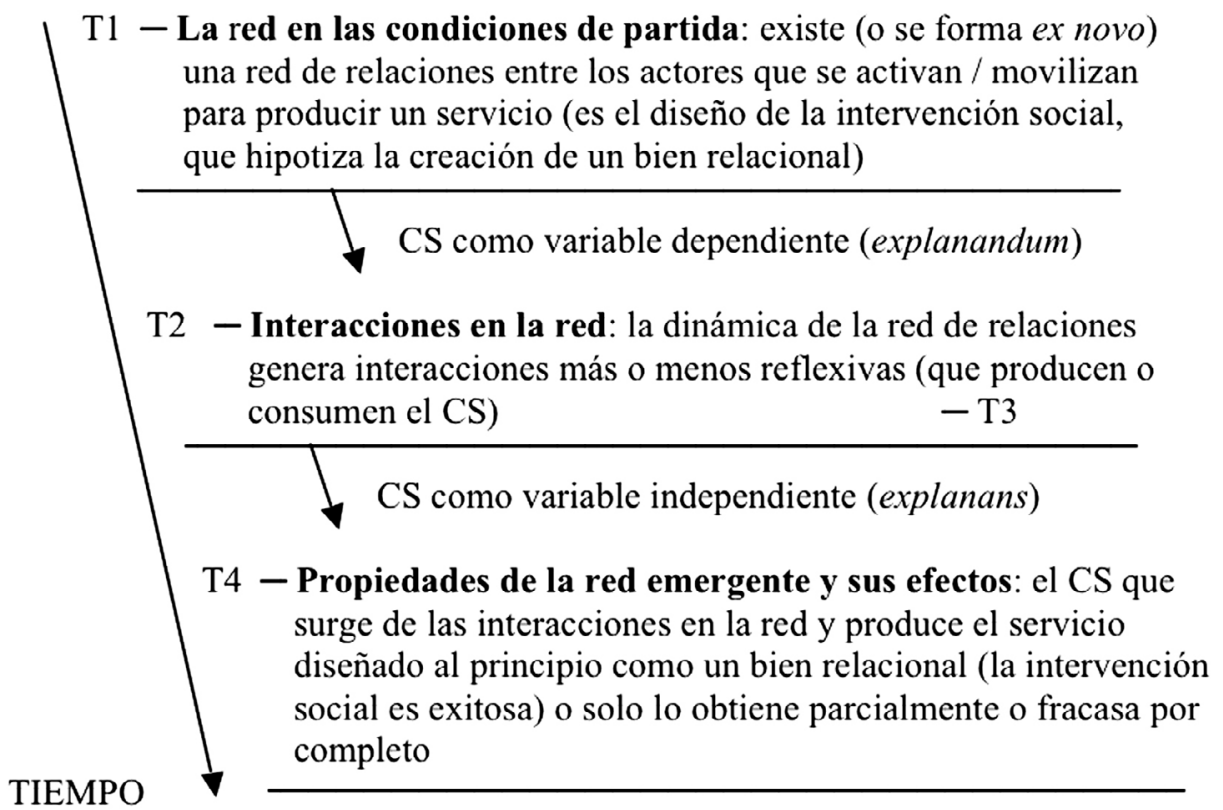

(un nuevo ciclo comienza)

El VSA es la diferencia entre la situación inicial en el tiempo T1 y la situación al final de ciclo morfogenético, considerado en el tiempo T4. La diferencia puede ser positiva, cuando hay un valor añadido (added value), o negativa, cuando hay un valor restado (subtracted value). ${ }^{6}$ El VSA da una medida de la capacidad de una red asociativa para ser eficaz qua talis -en consiguiente, no en relación con ningún otro elemento, sino por sí mismaen cuanto da medida de la capacidad de una red para producir sociabilidad como un valor añadido; es decir, un incremento de sus parámetros relacio-

6 En esta contribución me concentro en el valor añadido, pero muchas investigaciones indican que la dinámica de la red puede disminuir el valor en lugar de aumentarlo. Por ejemplo, en algunas asociaciones voluntarias, los voluntarios dicen que su confianza en otros miembros ha disminuido con el tiempo (Donati \& Colozzi, 2007). Dada la longitud limitada del texto, no puedo examinar los casos de valor disminuido. 
nales. Por ejemplo, el grado de reciprocidad, cooperación, confianza, afinidad, etc. En otras palabras, da una medida de si, y en qué grado, la red tiene éxito a la hora de poner su bien relacional -interno- en sinergia con el CS -en todas sus dimensiones: vínculos, puentes, enlaces- ya que unos necesitan a otros para producir los bienes de la pro-socialidad.

Podríamos encontrar un ejemplo en la figura de la Tagesmütter -la llamada day-mother. En el momento que se crea esta figura mediante un contrato, en el que se acuerda que una madre de un niño pequeño - de 0 a 3 años de edad-toma a su cuidado a otros -dos o tres- niños de la misma edad, se crea una relación entre las familias implicadas, que han acordado un mismo objetivo o tarea común: el cuidado de los niños, confiado a la tagesmütter. En esta acción de confianza y cooperación recíproca se da la premisa -y la promesa- de un bien relacional. Depende de la segunda fase -de cómo vayan las interacciones entre las familias- asegurar que las interacciones entre ellos generen, y no consuman, CS; en otras palabras, si se crea un contexto relacional construido en la confianza, la cooperación y la reciprocidad entre los padres de los niños -los cuales, en función del tipo de relación que se establezca, se conocen y pasan tiempo juntos en contacto con cualidades y capacidades que una guardería, pública o privada, organiza de una manera burocrática o comercial, no podrían producir, entonces el CS genera una red de familias en la que florecen los bienes relacionales. De otro modo, la intervención social toma un camino bien distinto.

En general, el llamado tercer sector y el sector social privado son tales cuando se configuran como esferas sociales que producen inclusión social relacional mediante la interacción virtuosa de los bienes relacionales y el CS. La interacción es virtuosa tanto porque los hace crecer, como por operar con fines pro-sociales, cívicos o civiles. La «economía cordial» de la que Patrici Calvo (2018) habla es una posibilidad.

La conceptualización presentada aquí -Figura 2- resuelve las aporías que mantenían a los estudios sobre el CS cautivos -en concreto, los citados por autores como Portes (1998), Edwards et al. (eds. 2001)- en cuanto demuestra que el CS es una variable que es tanto dependiente como independiente. Dependiente del contexto, así como de la actividad, de la cultura -las normas de los actores, los valores, las actitudes-, así como de la estructura -hecha de redes, organizaciones y conexiones-, sin confundir estos elementos y dimensiones. Se necesitaría un libro entero para discutir esto en profundidad; sin embargo, dado que el núcleo teórico de la cuestión está suficientemente claro, el presente trabajo tiene el objetivo de mostrar 
que el marco conceptual recién delineado se ve corroborado por la investigación empírica, cuyos resultados se muestran aquí de manera sintética.

\section{LA SOLUCIÓN AL PROBLEMA RADICA EN COMPRENDER EL VALOR SOCIAL AÑADIDO DE LAS RELACIONES SOCIALES}

En resumen, decir «valor añadido» significa hacer referencia al aumento de valor de algo/alguien; un incremento producido por algo/alguien que ha actuado/operado sobre algo/alguien que -debido a un efecto causal- ha visto aumentado su valor.

Cuando esto ocurre a través de la relación social, nos encontramos con el VSA de la relación social. Es la relación social la que da valor a algo/alguien. Si lo que da valor es una relación, entonces estamos produciendo un bien relacional, que será público si, y sólo si, permite el acceso y la posibilidad de adhesión de todos los que tengan un interés potencial en compartirlo como una forma de servicio relacional. Aquí el adjetivo público no es sinónimo de estatal, sino de un bien que está en el ámbito de las relaciones públicas y es accesible para todos aquellos que quieran participar en su producción y disfrute con otros miembros.

Para comprender la relación entre el bien público y el bien relacional, es necesario distinguir entre bienes públicos estatales y no estatales. Los bienes públicos no estatales tienen la característica de ser administrados por sus miembros de manera privada, pero no con fines de lucro, porque tienen fines de solidaridad social y por esta razón también se los llama 'bienes colectivos relacionales' o asociaciones sociales privadas (que practican la responsabilidad social).

La Figura 3 aclara las diferencias entre los diversos tipos de bienes producidos en la sociedad. La tipología se construye clasificando los bienes sociales sobre la base de dos ejes: según si el consumidor es soberano/no soberano y si el consumo es competitivo/no competitivo. De esta forma, los bienes privados se conciben como aquellos caracterizados por un consumidor soberano y un consumo competitivo (recuadro A). Los bienes públicos estatales, en cambio, son aquellos caracterizados por un consumidor no soberano y un consumo no competitivo (recuadro $G$ ), mientras que en el tercer recuadro (I), consumidor no soberano y consumo competitivo, encontramos los bienes relacionales «secundarios»-es decir, asociativos- o colectivos en la esfera pública de la sociedad civil. En el cuarto recuadro 
(L) se encuentran los bienes con consumidor soberano y consumo no competitivo, que son identificables en redes informales y familiares. ${ }^{7}$

Figura 3

Los cuatro tipos básicos de bienes producidos en la sociedad (bienes estatales, bienes relacionales en la esfera pública, bienes relacionales en las redes informales y familiares, y bienes estrictamente privados) (Donati, 2000)

\begin{tabular}{|c|c|c|}
\hline & Consumo no competitivo & Consumo competitivo \\
\hline $\begin{array}{l}\text { Consumidor no } \\
\text { soberano }\end{array}$ & $\begin{array}{l}\text { Bien público estatal } \\
\text { ( } G=\text { estado })\end{array}$ & $\begin{array}{c}\text { Bien relacional secundario o } \\
\text { colectivo en la esfera pública } \\
\text { (I = Tercer sector } \\
\text { y Economía social })\end{array}$ \\
\hline $\begin{array}{l}\text { Consumidor } \\
\text { soberano }\end{array}$ & $\begin{array}{l}\text { Bien relacional primario } \\
\text { ( } \mathrm{L}=\text { cuarto sector: redes } \\
\text { informales y familiares })\end{array}$ & $\begin{array}{l}\text { Bien privado } \\
(\mathrm{A}=\text { Mercado })\end{array}$ \\
\hline
\end{tabular}

Una red asociativa, o una organización del tercer sector, y en general de la economía social (Herrero-Blasco 2014), crea VSA porque, al producir bienes o servicios -por ejemplo, un servicio de cuidado de niños, discapacitados, ancianos, etc. -, hace uso de un mayor número, y de mejor calidad, de relaciones sociales que las que utiliza el mercado o la burocracia de la administración pública. Si estas relaciones, más numerosas y de mejor calidad, tienen como objetivo, además de los simples servicios en cuanto tales, las relaciones entre los sujetos involucrados en ese servicio, entonces el servicio, en sí mismo, se convierte en un bien relacional. Se convierte en un servicio relacional en el sentido estricto.

Decir que una relación social tiene un VSA significa tomar nota del hecho de que poner a algo/alguien en relación con otro algo/alguien produce una entidad que va más allá de las capacidades y cualidades de los elementos/sujetos que se ponían en relación. La frase "poner dos entidades en relación» significa tanto que una entidad hace referencia simbólica a la otra -refero- como que se ha creado un vínculo o una conexión estructural -religo.

7 Las letras A, G, I, L se refieren al esquema AGIL revisado en clave relacional: Donati (2006). 
La combinación relacional de la referencia simbólica y el vínculo estructural -refero-religo, que puede llamarse «relación fundadora»-o Grundverbältnis en alemán (Günther, 1976: 349), genera un valor añadido porque aumenta el valor de lo que sea que se ponga en relación a través de la relación social misma. Éste es un efecto emergente, y un efecto que surge precisamente de las cualidades de la relación y de sus propiedades causales.

Para poder entender este proceso de valorización, es necesario conceptualizar la 'relación que valoriza, dando que no todas las relaciones tienen este efecto -el de producir bienes relacionales. En ocasiones, el efecto que surge es negativo, en el sentido de que se produce un mal relacional (MR) en lugar de un bien relacional (BR). Así, surge la siguiente cuestión: ¿Cómo se configura una relación que da valor añadido?

Lo vemos en la Figura 4. El valor de algo/alguien puede definirse -de acuerdo con el marco teórico AGIL en su versión relacional- según cuatro dimensiones, que están interrelacionadas entre sí: como valor de cambio (A), como valor de uso (G), como valor relacional o valor del vínculo (I) y como valor de dignidad (L).

(A) Se valora a algo/alguien en términos de valor de cambio cuando, a través de la relación con algo/alguien distinto de sí mismo, su valor económico -Wert- se ve incrementado con referencia al sistema de precios -valor añadido en términos de utilidad.

(G) Se valora a algo/alguien en términos de valor de uso cuando, a través de la relación con algo/alguien distinto de sí mismo, sus usos posibles se ven incrementados. En otras palabras, se genera una super-funcionalidad de algo/alguien -valor añadido en términos de funciones/servicios ofrecidos.

(I) El valor relacional es el valor de algo/alguien debido a las relaciones que hace posibles, o debido a las relaciones que activa o que son necesarias para configurar cada una de las otras dimensiones y sus combinaciones para mejorar las cualidades y capacidades de lo que sea -algo/alguien- que se haya puesto en relación -valor añadido en términos de creación de relaciones que mejoran las capacidades y cualidades de lo que se ha puesto en relación. Las relaciones que llamamos capital social -confianza, cooperación, reciprocidad- optimizan este criterio.

(L) Se valora a algo/alguien en su valor de dignidad cuando, a través de la relación con algo/alguien distinto de sí mismo, el sentido de 
dignidad que uno tiene para sí mismo se reconoce y se intensifica, sin posibilidad de negociar este valor -Würde en alemán; valor añadido en términos de reconocimiento.

Figura 4

Las dimensiones analíticas del «valor» de algo/alguien. El valor añadido- puede medirse como ventaja obtenida en el intercambio, en la utilidad de algo/alguien, en la dignidad de algo/alguien, en la relación social que se activa o se estimula por algo/alguien y que, como un vínculo activo, ofrece nuevas oportunidades y recursos relaciones que no son simplemente de intercambio o de uso

G

Valor de uso

(Dimensión política:

la potencial sobre-funcionalidad)

A

Valor de cambio

(dimensión económica:

valor en el sentido económico,

Wert, con referencia a un precio)

\author{
I \\ Valor relacional \\ (dimensión de enlace: \\ los recursos que la relación \\ activa empoderando a los sujetos)
}

$\mathrm{L}$

Valor como dignidad

(Wert entendido como Würde, es decir, como lo que es digno

en sí mismo y para sí mismo)

Éstas son las dimensiones analíticas de «valor». En los procesos sociales, el valor de algo/alguien puede verse incrementado, simplemente reproducido tal cual, disminuido o destruido. La valorización es una operación de mejora -en tanto que incrementar las cualidades de algo/alguien- que puede referirse a una de las cuatro dimensiones presentadas en la Figura 3 o a una combinación de ellas.

Cuando la gente moviliza una red de relaciones para mejorar el valor de algo/alguien -por ejemplo, en el área de servicios sociales-, la gente está tratando de producir, de manera más o menos consciente, un valor social añadido. Pero lo consiguen si, y sólo si, utilizan el criterio relacional, el cual 
no se refiere ni a la utilidad económica ni al servicio funcional, sino a la capacidad de las relaciones sociales de movilizar recursos -incluyendo las relaciones mismas- que empoderan a los sujetos que son, al mismo tiempo, productores y usuarios de los servicios (prosumers). Este tipo de operación es, en teoría, específico del sector del privado-social y del tercer sector, ${ }^{8}$ cuando dan prioridad a los criterios de valor que hacen referencia a las dimensiones relacional y de dignidad.

Para que esto ocurra, es necesario que la relación social que da un valor añadido a algo/alguien (X) se haga posible. Esto quiere decir que debe haber potencial para una cierta cantidad de capital social; esto es, una cierta disposición a tener relaciones de confianza, cooperación y reciprocidad. Estas relaciones pertenecen al área de valor como dignidad y valor relacional -área L-I-; es decir, al área de las relaciones que confieren un valor intrínseco a algo/alguien (X). Si esta área funciona bien, es posible producir un bien relacional que, por su parte, alimenta el capital social en una secuencia no circular, sino con carácter lineal de sucesión en el tiempo -sin posibilidad de ir en sentido contrario- como vimos en la Figura 2.

De este modo, la capacidad de relación que se despliega en la interacción entre los actores puede aumentar o decrecer según los parámetros del capital social -confianza, etc.- de los que dependen los bienes públicos relacionales. Si estos parámetros caen por debajo de un determinado umbral, o incluso caen hasta cero, no sólo no se produce el bien público relacional, sino que se consume el capital social. Esto es lo que sucede habitualmente si el proceso de valorización de algo/alguien permanece confinado en el área del valor de cambio - utilitario- o el valor de uso -área A-G-; esto es, el área de interacciones que da prioridad al valor extrínseco de la relación social. Vemos ocurrir todo esto en el nivel macro, meso y micro, tal como las investigaciones hasta este momento citadas han sostenido y demostrado.

Una nota final. En principio, el área del valor extrínseco -área A-G, como, por ejemplo, el capital de Marx y la acción estratégica de Habermas- y el área de valor intrínseco -área L-I, como, por ejemplo, la relación yo-tú de Buber y la acción comunicativa de Habermas- están en las antípodas -en la Figura 3. Sin embargo, en los procesos reales de valorización esa diferencia se da en un desarrollo, en función del tiempo -las fases temporales- y del asunto tratado, por lo que es aconsejable no dicotomizar las formas de

8 El privado-social es cualquier organización que sea autónoma y con gestión privada, pero con fines prosociales públicamente accountable. Por ejemplo, una asociación voluntaria o una empresa que sean socialmente responsables. Para mayor información al respecto, véase Donati \& Colozzi (2007). 
valorización. No debería asumirse, por ejemplo, que el valor relacional añadido, teniendo en y por sí mismo un carácter cualitativo propio, sea necesariamente incompatible con, digamos, el valor de cambio añadido, que es extrínseco. Por el contrario, las tendencias actuales de fomentar la cooperación entre las organizaciones con y sin ánimo de lucro, estableciendo nuevas entidades - por ejemplo, las fundaciones civiles- que se asocian para producir bienes relacionales, están dirigiéndose, precisamente, en la dirección de un proceso "compuesto» de valorización en el cual los cuatro criterios de valor - en la Figura 3- trabajan juntos para crear un valor social añadido «total».

\section{AGRADECIMIENTOS}

Me gustaría agradecer al profesor Patrici Calvo, Universitat Jaume I, sus consejos y comentarios sobre mi artículo, que me han sido muy útiles. Nuestras investigaciones personales tienen muchos puntos en común y especialmente comparten un sentimiento ético común sobre qué debe ser la investigación social.

\section{BIBLIOGRAFÍA}

Abad Montesinos, Jaime \& Abad Montesinos, Mercedes (2014). La economía social y solidaria como alternativa económica. Bienes comunes y democracia. Recerca. Revista de Pensament i Análisi, (15), 55-75.

Archer, Margaret S. (Ed) (2013). Social Morpbogenesis. Cham: Springer.

Ballet, Jérôme \& Guillon, Roland (Eds) (2003). Regards croisés sur le capital social. París: L'Harmattan.

Banfield, Edward C. (1958). The moral basis of a backward society. Nueva York: Free Press.

Becchetti, Leonardo; Pelloni,Alessandra \& Rossetti, Fiammetta (2008). Relational Goods, Sociability, and Happiness. Kyklos. International Review Social Science, 61(3), 343-363.

Bruni, Luigino (2005). Felicità, economia e beni relazionali. Nuova Umanità, 27(2-3), 537-559.

Bidart, Claire (1997). L’amitié. Un lien social. París: La Découverte.

Borgatti, Stephen P; Jones, Candace \& Everett, Martin G. (1998). Network Measures of Social Capital. Connections, 21(2), 27-36. 
Burt,Ronald S.(1995).Le capital social,les trous structuraux et l'entrepreneur. Revue française de sociologie, XXXVI(4), 599-628.

Burt, Ronald S. (2000). The Network Structure of Social Capital. En Sutton, Robert I. \& Staw, Barry M. (Eds). Research in Organizational Behavior (345-423). Greenwich, CT: JAI Press.

Burt, Ronald S. (2001). Structural Holes versus Network Closure as Social Capital. En Lin, Nan; Cook, Karen \& Burt, Ronald S. (Eds). Social Capital. Theory and Research (31-56). Nueva York:Aldine de Gruyter.

Burt, Ronald S. (2005). Brokerage and Closure. An Introduction to Social Capital. Nueva York: Oxford University Press.

Calvo, Patrici (2018). The Cordial Economy - Ethics, Recognition and Reciprocity. Cham: Springer.

Cartocci, Roberto (2007). Mappe del tesoro.Atlante del capitale sociale in Italia. Bolonia: Il Mulino.

Coleman, James S. (1988). Social Capital in the Creation of Human Capital. American.Journal of Sociology [Supplement], (94), 95-120.

Colozzi, Ivo (2005). Cosa sono i beni relazionali: un confronto fra approcci e approccio sociologico. Sociologia, 39(2), 13-20.

Colozzi, Ivo (2006). Terzo settore e valutazione di qualità: misurare la produzione di beni relazionali. Lavoro sociale, 6(3), 411-419.

Degenne, Alain \& Forsé, Michel (2003). The Concept of Social Capital. A Consideration of a Definition and an Empirical Application [Working Paper, Lasmas-CNRS-Ehess]. Caen: Université de Caen.

Donati, Pierpaolo (1989). Nuove istanze sociali e dignità umana. En Donati, Pierpaolo (Ed). La cultura della vita. Dalla società tradizionale a quella post-moderna (161-182), Milán: FrancoAngeli.

Donati, Pierpaolo (2000). La cittadinanza societaria. Roma-Bari: Laterza.

Donati, Pierpaolo (Ed) (2003). Famiglia e capitale sociale nella società italiana. Cinisello Balsamo: Edizioni San Paolo.

Donati, Pierpaolo (2006). L'analisi relazionale: regole, quadro metodologico, esempi. En Donati, Pierpaolo (Ed). Sociologia. Una introduzione allo studio della società (195-251), Padua: Cedam.

Donati, Pierpaolo (2010). Reflexivity after Modernity: From the Viewpoint of Relational Sociology. En Archer, Margaret S. (Ed). Conversations About Reflexivity (144-164). Londres \& Nueva York: Routledge.

Donati, Pierpaolo (2011). Relational Sociology. A New Paradigm for the Social Sciences. Londres \& Nueva York: Routledge.

Donati, Pierpaolo \& Calvo, Patrici (2014). New Insights into Relational Goods. Recerca. Revista de Pensament $i$ Anàlisi, (14), 7-17. 
Donati, Pierpaolo \& Colozzi, Ivo (Eds) (2006). Capitale sociale delle famiglie e processi di socializzazione. Un confronto fra scuole statali e di privato sociale. Milán: FrancoAngeli.

Donati, Pierpaolo \& Colozzi, Ivo (Eds) (2007). Terzo settore, mondi vitali e capitale sociale. Milán: FrancoAngeli.

Donati, Pierpaolo \& Solci, Riccardo (2011). I beni relazionali; che cosa sono e quali effetti producono. Torino: Bollati Boringhieri.

Donati, Pierpaolo \& Tronca, Luigi (2008). Il capitale sociale degli italiani. Le radici familiari, comunitarie e associative del civismo. Milán: FrancoAngeli.

Edwards, Bob; Foley, Michael W. \& Diani, Mario (2001). Beyond Tocqueville: Civil Society and the Social Capital Debate in Comparative Perspective. Hanover-Londres: University Press of New England,Tufts University. Fragkandreas, Thanos (2012). Reflections on social capital and economic performance. International Review of Sociology, 22(2), 259-271.

Gui, Benedetto \& Sugden, Robert (Eds) (2005). Economics and Social Interaction. Accounting for Interpersonal Relations. Cambridge: CUP.

Günther, Gotthard (1976). Formal Logic, Totality and the Super-Additive Principle. En Günther, Gotthard. Beiträge zur Grundlegung einer operationsfäbigen Dialektik (329-351). Hamburg: Felix Meiner.

Herrero-Blasco, Aurelio (2014). La Economía Social: su función económica y las políticas públicas de fomento. Recerca. Revista de Pensament $i$ Anàlisi, (15), 77-92.

Lin, Nan (1999). Building a Network Theory of Social Capital?. Connections, 22(1), 28-51.

Lin, Nan (2000). Inequality in Social Capital. Contemporary Sociology, 29(6), 785-795.

Lin, Nan (2001a). Social Capital. A Theory of Social Structure. Nueva York: Cambridge University Press.

Lin, Nan (2001b). Building a Network Theory of Social Capital. En Lin, Nan; Cook, Karen \& Burt, Ronald S. (Eds). Social Capital. Theory and Research (3-29). Nueva York: Aldine de Gruyter.

Portes, Alejandro (1998). Social Capital: Its Origins and Applications in Modern Sociology. Annual Review of Sociology, (24), 1-24.

Putnam, Robert D. (1993). Making Democracy Work: Civic Traditions in Modern Italy. Princeton, NJ: Princeton University Press.

Putnam, Robert D. (2000). Bowling Alone. The Collapse and Revival of American Community. Nueva York: Simon \& Schuster. 
Ramis Olivos, Álvaro (2014). Los bienes comunes intangibles en el capitalismo cognitivo. Recerca. Recerca. Revista de Pensament i Anàlisi, 15, 109-129.

Rossi, Giovanna \& Boccacin, Lucia (2006a) Le forme e l'esito societario del capitale sociale in una organizzazione multilivello di terzo settore. En Donati, Pierpaolo \& Colozzi, Ivo (Eds). Terzo settore e valorizzazione del capitale sociale in Italia: luoghi e attori. Milán: FrancoAngeli.

Rossi, Giovanna \& Boccacin, Lucia (2006b). Il capitale sociale in un'organizzazione multilivello di terzo settore. Milán: FrancoAngeli.

Sacco, Pier Luigi \& Vanin, Paolo (2000). Network Interaction with Material and Relational goods: an Exploratory simulation. Annals of Public and Cooperative Economics, 71(2): 229-259.

Sacco, Pier Luigi \& Zamagni, Stefano (eds.). (2006). Teoria economica e relazioni interpersonali, Bolonia: il Mulino.

Uhlaner, Carole J. (1989). 'Relational Goods' and Participation. Incorporating Sociability into a Theory of Rational Action. Public Choice, (62): 253-285.

Uhlaner, Carole J. (2014). Relational Goods and Resolving the Paradox of Political Participation. Recerca. Revista de Pensament i Anàlisi, 14: 47-72.

Uslaner, Eric M. (2002). The Moral Foundations of Trust. Cambridge, Mss.: Cambridge University Press.

Wollebaek, Dag \& Selle, Per (2002). Does Participation in Voluntary Associations Contribute to Social Capital? The Impact of Intensity, Scope and Type. Nonprofit and Voluntary Sector Quarterly, 31(1), 32-61. 